\title{
Endothelial-like cells differentiated from mesenchymal stem cells attenuate neointimal hyperplasia after vascular injury
}

\author{
JIANZHONG XU, DUOJIAO WU, YAN YANG, KAIDA JI and PINGJIN GAO \\ State Key Laboratory of Medical Genomics, Shanghai Key Laboratory of Vascular Biology, Department of Hypertension, \\ Shanghai Institute of Hypertension, Ruijin Hospital, Shanghai Jiaotong University School of Medicine, \\ Shanghai 200025, P.R. China
}

Received September 9, 2015; Accepted September 21, 2016

DOI: $10.3892 / \mathrm{mmr} .2016 .5799$

\begin{abstract}
The present study investigated the contribution of bone marrow-derived mesenchymal stem cells (BM-MSCs) to neointimal formation, and whether endothelial-like cells (ELCs) differentiated from BM-MSCs could attenuate intimal hyperplasia following vascular injury. BM-MSCs were isolated from rat femurs and tibias and expanded ex vivo. Differentiation into ELCs was induced by cultivation in the presence of $50 \mathrm{ng} / \mathrm{ml}$ vascular endothelial growth factor (VEGF). MSCs and ELCs were labeled with BrdU and injected via the femoral vein on the day of a balloon-induced carotid artery injury. Carotid artery morphology and histology were examined using ultrasound biomicroscopy and immunohistochemistry. Flow cytometry analysis measured CD31 and CD34 expression, and immunofluorescence analysis measured von Willebrand factor and VEGF receptor 2 expression in ELCs. Ultrasound biomicroscopy observed a significantly increased intima-media thickness in the phosphate-buffered saline (PBS) and BM-MSCs groups compared with the ELCs group. Intima/media ratios were significantly reduced in the ELCs group compared with the PBS and BM-MSCs groups. At 4 weeks of administration, the cells labeled with BrdU were abundantly located in the adventitial region and neointima. MSCs were able to differentiate into ELCs in vitro. Cell therapy with BM-MSCs was not able to attenuate neointima thickness, however transplantation with ELCs significantly suppressed intimal hyperplasia following vascular injury.
\end{abstract}

Correspondence to: Dr Jianzhong Xu or Dr Pingjin Gao, State Key Laboratory of Medical Genomics, Shanghai Key Laboratory of Vascular Biology, Department of Hypertension, Shanghai Institute of Hypertension, Ruijin Hospital, Shanghai Jiaotong University School of Medicine, 2 Ruijin Road, Shanghai 200025, P.R. China

E-mail: jianzhongxv@outlook.com

E-mail: gaopingjin@yahoo.com.cn

Key words: mesenchymal stem cells, endothelial-like cells, neointimal hyperplasia

\section{Introduction}

Cellular therapy has been widely researched, and is commonly used to treat cardiovascular disease. Bone marrow-derived mesenchymal stem cells (BM-MSCs) are nonhematopoietic multi-potent stem cells and have an intrinsic ability to differentiate into functional cell types able to repair diseased or injured tissue (1). BM-MSCs can be readily obtained, rapidly proliferate in culture, and display a capacity to differentiate towards endothelial $(2,3)$ or vascular smooth muscle cells (VSMCs) (4), and they serve an important role in postnatal neovascularization in various tissue contexts. Neointimal hyperplasia in response to arterial injury is a complex process, and it has been previously suggested that neointimal lesions also contain BM-MSCs attracted to the vascular injury site (5). However, the effect of BM-MSCs in vascular injury remains to be fully elucidated. Certain studies have demonstrated the capacity of BM-MSCs to restoring the endothelial lining and reduce neointimal formation following injury (6-8). However, contradictory studies have demonstrated that the transplantation of BM-MSCs was unable to reduce neointimal hyperplasia (9), and potentially aggravated neointimia formation $(10,11)$.

It is widely accepted that BM-MSCs can be induced to differentiate toward endothelial-like cells (ELCs) in vitro (2). In the present study, a method for the isolation, growth and ex vivo expansion of ELCs differentiated from BM-MSCs was described, and it was hypothesized that ELCs could attenuate neointimal hyperplasia following arterial injury.

\section{Materials and methods}

Animals. The animal use in this study was approved by the Animal Care and Use Committee of Shanghai Jiaotong University School of Medicine (Shanghai, China), and all procedures were conducted in accordance with institutional guidelines. A total of 12 young male Sprague-Dawley (SD) rats (weight, 100-150 g; age, 4 weeks) were used to isolate the MSCs. A total of 26 adult male SD rats (weight, 300-350 g; age, 16 weeks) were used in the carotid balloon-injury model and cell transplantation experiments. Rats were given ad libitum access to food and water, unless otherwise specified. The rats were maintained under controlled temperature $\left(20-24^{\circ} \mathrm{C}\right)$, 
humidity (30-70\%) and lighting conditions (12:12 h ligh/dark cycle). Subsequently, rats were sacrificed following abdominal injection of $10 \%$ chloral hydrate $(600 \mathrm{mg} / \mathrm{kg})$.

MSC isolation and culture. BM-MSCs were collected from the bone marrow of young male SD rat femurs and tibias, as previously described (12). The femoral and tibial bones were obtained from donor rats under anesthesia with $10 \%$ chloral hydrate $(300$ mg/kg; Sigma-Aldrich; Merck Millipore, Darmstadt, Germany). Bone marrow was flushed with low glucose Dulbecco's modified Eagle's medium (L-DMEM; Gibco; Invitrogen; Thermo Fisher Scientific, Inc., Waltham, MA, USA) containing $15 \%$ (v/v) fetal bovine serum (FBS; Gibco; Invitrogen; Thermo Fisher Scientific, Inc.), $100 \mathrm{U} / \mathrm{ml}$ penicillin and $100 \mathrm{U} / \mathrm{ml}$ streptomycin using a syringe with a 21-gauge needle. Cells from one rat were plated into two $35 \mathrm{~mm}$ dishes at $37^{\circ} \mathrm{C}$ in a humidified atmosphere of $5 \% \mathrm{CO}_{2}$. Non-adherent cells were removed by changing the medium after $48 \mathrm{~h}$. The cells were then incubated for $5-7$ days at $37^{\circ} \mathrm{C}$ in a humidified atmosphere to reach confluence. Once the cells had grown to near confluence, they were passaged two to three times, being detached with $0.25 \%$ trypsin/1 mM EDTA, and were re-plated at a density of $1 \times 10^{6} / \mathrm{ml}$. Finally, MSCs were propagated for 3-6 passages for further experiments.

BM-MSCs differentiated into ELCs. Confluent cells were cultivated in the presence of endothelial cell growth medium-2 (EGM-2; Cambrex Corporation, East Rutherford, NJ, USA) with $2 \% \mathrm{FBS}$ and $50 \mathrm{ng} / \mathrm{ml} \mathrm{rVEGF164}$ (R\&D Systems, Inc., Minneapolis, MN, USA) for 7 days. The medium was changed every 2 days. Cells were propagated for 3-6 passages after 7 days.

Flow cytometric analysis of MSCs and ELCs. Cells were trypsinized, washed with phosphate-buffered saline (PBS), and incubated with the following antibodies: Phycoerythrin (PE)-mouse anti-rat CD31 (cat. no. 555027), PE-mouse anti-rat CD54 (cat. no. 554970), PE-mouse anti-rat CD71 (cat. no. 554891), PE-mouse anti-rat CD90 (cat. no. 554898), PE-Cy ${ }^{\mathrm{TM}} 5$ mouse anti-rat CD45 (cat. no. 559135), and fluorescein isothiocyanate (FITC)-mouse anti-rat CD34 (cat. no. 555821; BD Biosciences, Franklin Lakes, NJ, USA). Analysis was performed using a FACSCalibur flow cytometer (BD Biosciences).

Fluorescence immunocytochemistry. Immunofluorescence analysis was used to detect the expressions of ELC surface antigens von Willebrand factor (vWF) and vascular endothelial growth factor (VEGF) receptor 2 (R2). Cultured MSCs grown in slide chambers were fixed in $4 \%$ buffered paraformaldehyde for $10 \mathrm{~min}$, incubated with blocking solution [2\% bovine serum albumin (BSA; Sigma-Aldrich; Merck Millipore)] for $1 \mathrm{~h}$ at room temperature, and then incubated with the primary antibody (mouse anti-rat vWF, cat. no. sc-365712, 1:50; mouse anti-rat VEGFR2, cat. no. sc-393179, 1:50; Santa Cruz Biotechnology, Inc., Santa Cruz, CA, USA) in blocking solution overnight at $4^{\circ} \mathrm{C}$. Negative controls were incubated with blocking solution only. The samples were subsequently incubated with monoclonal anti-mouse FITC-conjugated secondary antibodies (1:100; cat. no. sc-358943; Santa Cruz
Biotechnology, Inc.) for $1 \mathrm{~h}$ at room temperature. The nuclei were stained with Hoechst 33,258 (Sigma-Aldrich; Merck Millipore) for $5 \mathrm{~min}$ and the samples examined under a fluorescence microscope (Leica Microsystems GmbH, Wetzlar, Germany).

Cell labeling. The third and sixth passaged cells were collected and labeled with 5-bromo-2-deoxyuridine (BrdU; Sigma-Aldrich; Merck Millipore,). The cells were incubated in complete medium with $20 \mu \mathrm{mol} / 1 \mathrm{BrdU}$ to label cells in the $\mathrm{S}$ phase of the cell cycle during a $48 \mathrm{~h}$ period.

Rat carotid balloon-injury model and cell transplantation. Adult SD rats were anesthetized with $10 \%$ chloral hydrate (300 mg/kg) and underwent a balloon catheter injury to the right carotid artery, as previously described (13). A 2F Fogarty balloon catheter (Edwards Life Sciences Corporation, Irvine, CA, USA) was inserted through the right external carotid artery. The balloon was inflated with $0.1 \mathrm{ml}$ saline to distend the common carotid artery (CCA) and passed 3 times along the isolated segment length (1 to $1.5 \mathrm{~cm}$ in length), and then the catheter was removed.

All rats $(n=18)$ were randomly divided into three groups. In group 1 (control), rats were given PBS alone (without donor cell administration) injected on the day of the carotid injury operation, immediately subsequent to balloon injury $(n=6)$. In group 2, rats were administered with rat MSCs $\left(2 \times 10^{6} / \mathrm{ml}\right)$ in $1 \mathrm{ml}$ of total fluid volume (L-DMEM) injected through the femoral vein $(n=6)$. In group 3 , rats were administered with ELCs $\left(2 \times 10^{6} / \mathrm{ml}\right)$ that were injected through the femoral vein $(n=6)$.

Ultrasound biomicroscopy imaging. An ultrasound biomicroscopy (UBM) system (Vevo 770; VisualSonics, Inc., Toronto, Canada) equipped with a $40 \mathrm{MHz}$ transducer was used for all examinations. The current study involved 18 rats, and UBM was performed on days 7, 14 and 28 subsequent to vascular injury. The same researcher conducted all examinations and performed the measurements in a blinded fashion.

Tissue processing and histological analysis. A total of 28 days subsequent to injury, the CCA was excised on both sides (injured and non-injured contralateral side artery). Each CCA was fixed with $10 \%$ buffered formalin and embedded in paraffin. Sections $5 \mu \mathrm{m}$ thick were taken from the middle portion of the balloon-injured segment, stained with a Masson's trichrome stain to evaluate general morphology, and photographed at a magnification of x100. Morphometric analyses of the digital images were performed using Leica QWin V3 software (Leica Microsystems $\mathrm{GmbH}$ ). The intima and media areas were determined, and the ratio between these two areas was calculated.

Immunohistochemistry and immunofluorescence. In order to detect the donor cells ex vivo, sections were dried at $60^{\circ} \mathrm{C}$ for $20 \mathrm{~min}$, deparaffinized and dehydrated, and treated with $0.3 \%$ hydrogen peroxide in methanol to block endogenous peroxidase activity. Subsequent to washing with PBS, tissues were incubated with BSA (5\%) at room temperature for $30 \mathrm{~min}$, then 
incubated with anti-BrdU (1:100; cat. no. ab125306; Abcam , Cambridge, UK) at $4^{\circ} \mathrm{C}$ overnight. Slides were rinsed in PBS, incubated in horseradish peroxidase-conjugated anti-mouse IgG (1:100; cat. no. sc-2373; Santa Cruz Biotechnology, Inc.) in BSA (1\%) for $1 \mathrm{~h}$, stained with 3,3'-diaminobenzidine, and analyzed using a Zeiss Axioskop 50 light microscope (Carl Zeiss AG, Oberkochen, Germany).

To detect donor cell differentiation in vivo, endothelial cells and VSMCs were identified using immunostaining with mouse anti-rat CD31 antibody (1:50; cat. no. sc-52713; Santa Cruz Biotechnology, Inc.) and rabbit anti- $\alpha$-smooth muscle actin ( $\alpha$-SMA) antibody (1:100; cat. no. ab15734; Abcam), respectively. Nuclear staining was conducted using anti-BrdU. Slides were pre-incubated with $5 \%$ BSA for 30 min each. The primary antibody was then applied at $4^{\circ} \mathrm{C}$ overnight, followed by application of the appropriate tetramethylrhodamine-conjugated IgG (1:200 dilution; cat. no. sc-3827; Santa Cruz Biotechnology, Inc.) and FITC-conjugated IgG (1:200 dilution; cat. no. sc-358943; Santa Cruz Biotechnology, Inc.). Sections were washed with PBS and then mounted at room temperature.

Statistical analysis. All results were expressed as the mean \pm standard deviation. Statistical significance was evaluated by one-way analysis of variance followed by Bonferroni post-hoc tests. $\mathrm{P}<0.05$ was considered to indicate a statistically significant difference. All statistical analyses were performed using SPSS software, version 13 (SPSS, Inc., Chicago, IL, USA).

\section{Results}

Cultured BM-MSCs can differentiate to endothelial cell phenotypes. BM-MSCs were cultured as plastic adherent cells in vitro, and were subsequently analyzed (Fig. 1). Their morphological features are presented in Fig. 1A. Characteristic flattened and spindle-shaped cells were recognized. Therefore, endothelial experiments were performed on cells from passage 3-6. Cells were tested with flow cytometry for the presence or absence of characteristic hematopoietic and endothelial markers. MSCs typically expressed the antigens CD54, CD71 and CD90. They were negative for the early hematopoietic marker CD34, endothelial cell marker CD31 and $8 \%$ expressed leukocyte marker CD45 (Fig. 1C).

Subsequent to 7 days of culture, the outgrowth cells cultivated by EGM-2 exhibited the typical 'cobblestone' endothelial cell morphology (Fig. 1B), and approximately $10 \%$ of cells expressed CD34 and CD31 (Fig. 1D). Immunofluorescence analysis indicated the expression of endothelial-specific markers such as vWF and VEGFR2 in ELCs, although the positive rate was less than $1 \%$ (Fig. 1E).

Ultrasound biomicroscopy imaging for detecting neointima formation. A comparative image series presented the neointimal site subsequent to balloon injury at 7,14 and 28 days, respectively (Fig. 2A). PBS (n=6), MSCs $\left(2 \times 10^{6} / \mathrm{ml}\right)(\mathrm{n}=6)$ and ELCs $\left(2 \times 10^{6} / \mathrm{ml}\right)(\mathrm{n}=6)$ were injected, respectively, through the femoral vein. After 4 weeks, the ELCs group attenuated intimal hyperplasia subsequent to vascular injury compared with the PBS and MSCs groups. Ultrasound biomicroscopy exhibited significant IMT increases in the PBS and BM-MSCs groups compared with the ELCs group at 14 days after arterial injury (0.14 $\pm 0.03 \mathrm{~mm}$ vs. $0.13 \pm 0.02 \mathrm{~mm}$ vs. $0.10 \pm 0.02 \mathrm{~mm} ; \mathrm{P}<0.01)$, and IMT was significantly increased at 28 days subsequent to injury $(0.23 \pm 0.04 \mathrm{~mm}$ vs. $0.22 \pm 0.05$ vs. $0.16 \pm 0.02 \mathrm{~mm}$; $\mathrm{P}<0.01$ ) (Fig. 2B).

Contribution of MSCs and ELCs to neointimal formation. Masson's trichrome-stained sections from the PBS, MSCs, and ELCs groups indicated that attenuated intimal hyperplasia was present in the ELCs group (Fig. 2C). The intima/media ratio was significantly reduced with therapy in the ELCs group $(1.22 \pm 0.02)$ when compared with the MSCs $(2.51 \pm 0.03)$ and PBS groups $(2.87 \pm 0.04)(\mathrm{P}<0.01)$. There was no significant difference between the MSCs and PBS groups following implantation ( $\mathrm{P}>0.05$; Fig. 2D).

Differentiation and homing of the MSCs and ELCs towards the site of injured carotids. Subsequent to intravenous transplantation of either BM-MSCs (Fig. 3C and D) or ELCs (Fig. 3E and F), the BrdU+- cells were identified at the site of injury on the arterial surface, predominantly in the adventitial area and particularly in the zone of the vasa vasorum, with few cells present in the neointima (Fig. 3). Numerous $\alpha$-SMA/BrdU+-cells were identified in the BM-MSCs group at 28 days subsequent to arterial injury (Fig. 3G; upper panel). However, when using CD31 as a marker of highly differentiated ELCs, few neointima cells were BrdU+-MSCs (Fig. 3G). When ELCs were transplanted, numerous CD31+/BrdU+-cells were present in the adventitia (Fig. 3G; lower panel), and few $\alpha$-SMA(+)/BrdU+-cells were observed.

\section{Discussion}

ELCs were derived from BM-MSCs their therapeutic effects in treating attenuating neointimal hyperplasia were demonstrated. A sufficient number of ELCs were consistently obtained to allow transplantation for ex vivo applications within 2 weeks of initial plating. Furthermore, these cells were demonstrated to be home towards injury sites and significantly reduce neointimal hyperplasia associated with balloon-induced carotid injury in rats.

BM-MSCs can be induced to differentiate toward ELCs in vitro and in vivo (3). In the present study, their ability to differentiate into ELCs was confirmed by treating them with EGM-2 and VEGF. Subsequent to a 7-day differentiation, it was identified that the treatment resulted in endothelial cell marker expression of CD31, vWF, VEGFR2 and the early hematopoietic marker CD34, whereas in other studies ELCs did not express CD31 under the same culture conditions (2). One previous study demonstrated that MSCs cultured in the same medium differentiated into high amounts of DiI-AcLDL-positive cells and enhanced the presence of endothelial cell markers, including vWF (90\%), vascular endothelial-cadherin-(60\%), and platelet endothelial cell adhesion molecule 1 (48\%) (14). All results demonstrated the ability of BM-MSCs to differentiate into ELCs under in vitro-induction conditions. ELCs were identified not to be mature endothelial cells due to expression of endothelial progenitor cell marker CD34. MSCs, due to their clear ex vivo proliferation and multipotent differentiation, are 

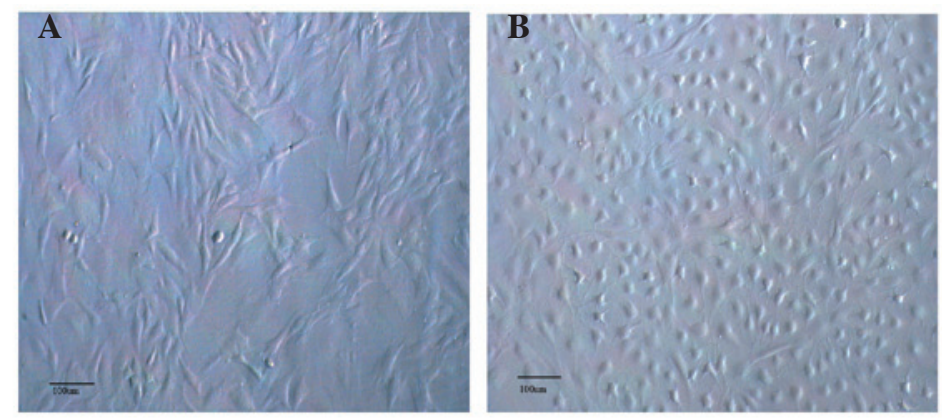

C
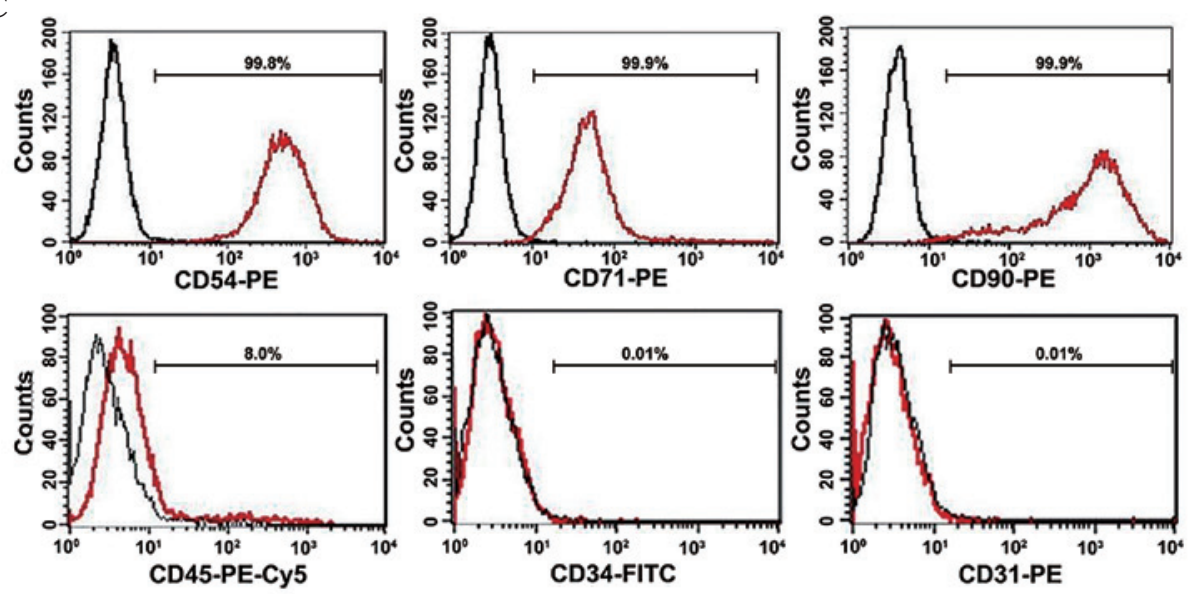

D
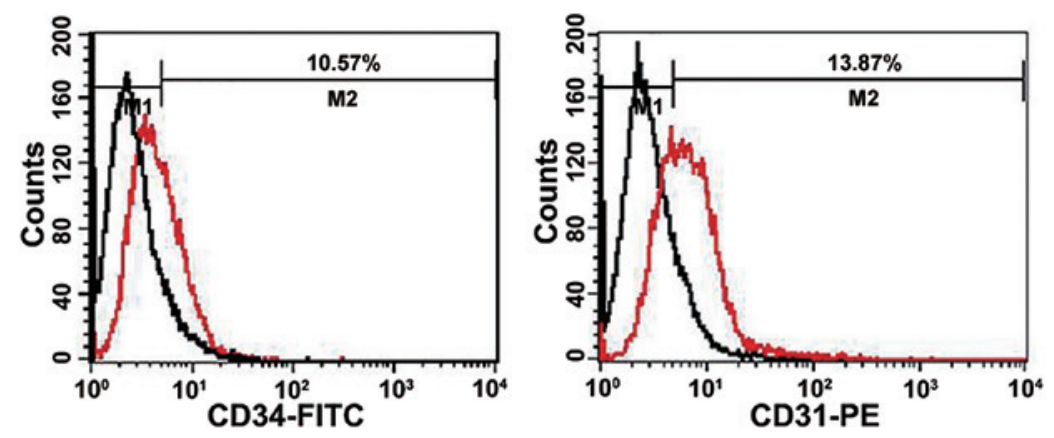

$\mathbf{E}$
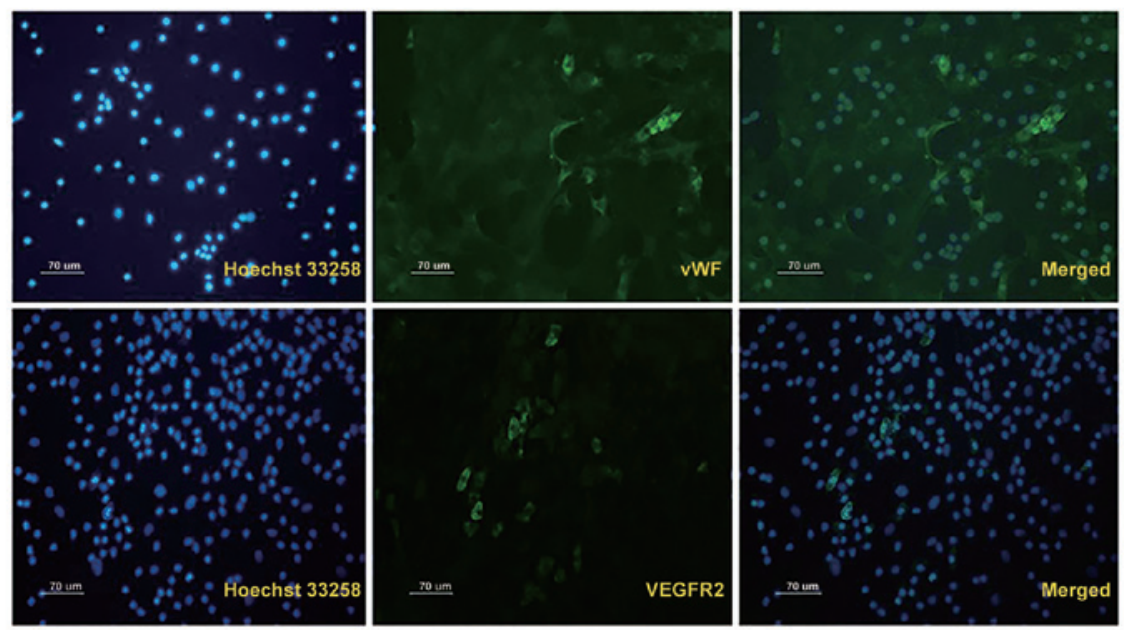

Figure 1. The morphological characteristics of (A) BM-MSCs and (B) ELCs (scale bar, $100 \mu \mathrm{m}$ ). Surface markers of (C) BM-MSCs and (D) ELCs analyzed by flow cytometry. (E) Immunofluorescence analysis highlighted the expression of endothelial-specific markers such as vWF and VEGFR2 in ELCs. BM-MSCs, bone marrow-derived mesenchymal stem cells; ELCs, endothelial-like cells; vWF, von Willebrand factor; VEGFR2, vascular endothelial growth factor receptor 2 .

ideal candidates for cell-based therapy (15) and may be a good potential option for ELCs to re-endothelialize following interventional procedures and tissue engineering.
In the current study, UBM, a highly feasible, non-invasive and simple technique to assess the neointima, was used. Previous studies reported that UBM-measured IMT, 
A

PBS

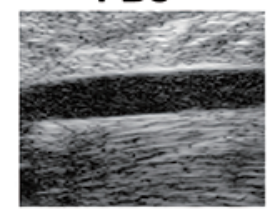

$14 \mathrm{~d}$

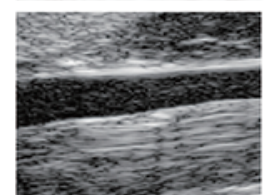

$28 \mathrm{~d}$

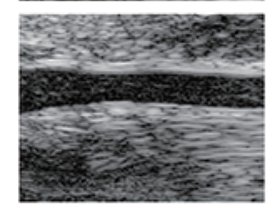

MSCs
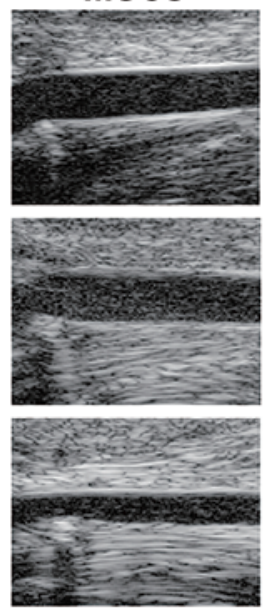

C

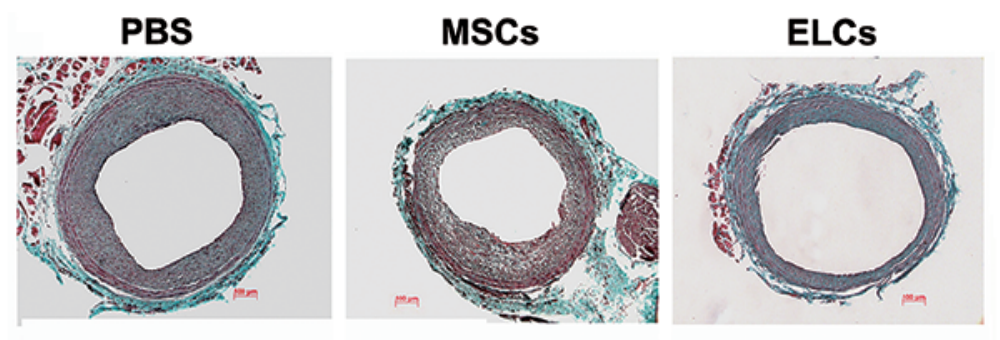

ELCs
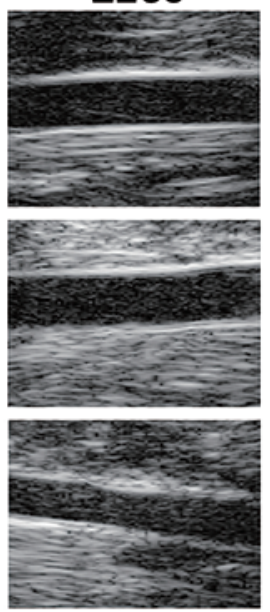

B

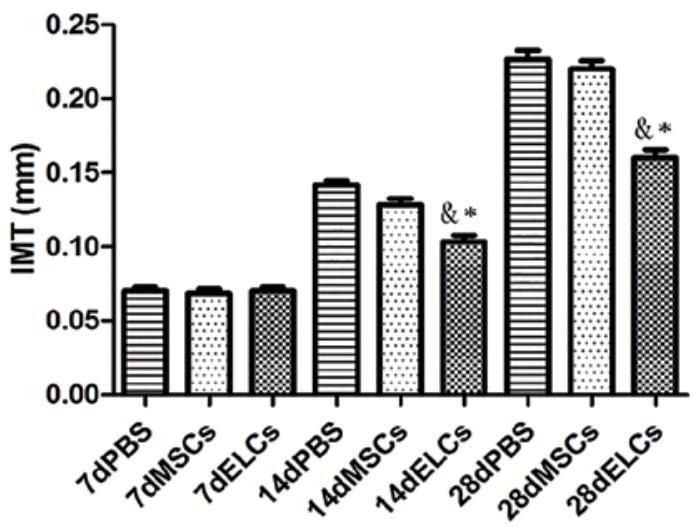

D

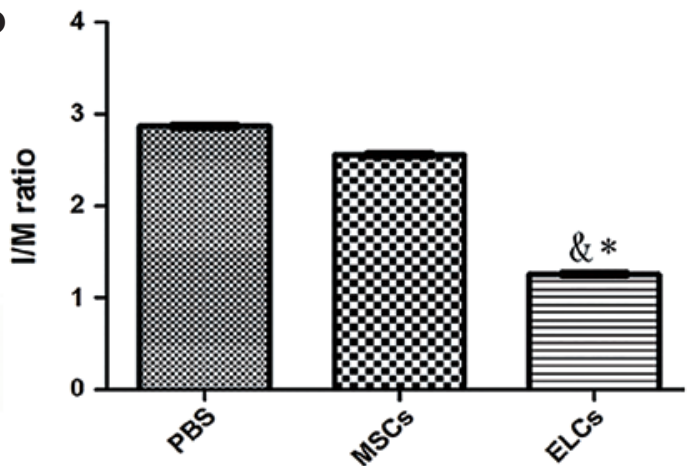

Figure 2. (A) A series of comparative images showing the site of the neointima subsequent to balloon injury at 7, 14, and 28 days. (B) PBS (n=6), MSCs $\left(2 \times 10^{6} / \mathrm{ml}\right)(\mathrm{n}=6)$ and ELCs $\left(2 \times 10^{6} / \mathrm{ml}\right)(\mathrm{n}=6)$ were injected through the femoral vein, respectively. Ultrasound biomicroscopy identified significant increases of IMT in the PBS and MSCs groups compared with the ELCs group 14 days subsequent to arterial injury $(0.14 \pm 0.03 \mathrm{~mm}$ vs. $0.13 \pm 0.02 \mathrm{~mm}$ vs. $0.10 \pm 0.02 \mathrm{~mm}$; $\mathrm{P}<0.01)$, and IMT significantly increased 28 days subsequent to injury $(0.23 \pm 0.04 \mathrm{~mm}$ vs. $0.22 \pm 0.05$ vs. $0.16 \pm 0.02 \mathrm{~mm}$; $\mathrm{P}<0.01)$. (C) Masson's trichrome staining for the contribution of MSCs and ELCs to neointimal formation. (D) The I/M ratio was significantly reduced in the ELCs group compared with the PBS and MSCs groups (1.22 \pm 0.03 vs. $2.52 \pm 0.04$ vs. $2.87 \pm 0.04 ; \mathrm{P}<0.001)(\mathrm{n}=6) .{ }^{*} \mathrm{P}<0.05$ vs. PBS group; ${ }^{\circledR} \mathrm{P}<0.05$ vs. MSCs group. PBS, phosphate-buffered saline; MSCs, bone marrow-derived mesenchymal stem cells; ELCs, endothelial-like cells; IMT, intima-media thickness; I/M, intima/media.

maximum plaque thickness and plaque area were highly correlated with histological data $(16,17)$. In the current study, UBM, which allowed for accurate real-time imaging of the continuously changing neointima in injured carotids was used, and no significant differences among the three groups were observed at 7 days. Between 7 and 14 days of age, growth in the neointima was observed. IMT in the ELCs group was significantly reduced compared with the PBS and BM-MSCs groups at 14 and 28 day subequent to injury, and appeared to increase in a time-dependent manner with maximal neointima at the injury artery identified after 28 days. This is, to the best of our knowledge, the first study to employ UBM real-time non-invasive assessment of the alterations of the injured artery, and identified that ELCs can attenuate the neointima.

The ability of MSCs to differentiate into either endothelial cells or VSMCs in vitro has been previously well characterized and documented (18-21). In comparison, differentiation to endothelial cells or vascular muscle cells in vivo subsequent to arterial injury remains unclear. Previous studies reported that transplanting BM-MSCs aggravates neointimal hyperplasia by affecting vasculature and differentiating into VSMCs $(18,19)$.
However, other studies indicate that engrafted MSCs appear to differentiate into endothelial cells, diminish neointimal formation and improve endothelial function $(20,21)$. BM-MSCs have two different effects on vascular cells subsequent to arterial injury, although their differentiated direction remains controversial. Thus, it is suggested that VEGF may be used to direct MSCs towards an endothelial lineage in vitro prior to transplantation in vivo. This is the first time, to the best of our knowledge, that ELCs derived from BM-MSCs in vitro were reported to reduce neointimal hyperplasia. The precise mechanism of ELCs remains unclear, however data from the current study indicated that ELCs can home to the surface of the injured vessel and re-endothelize it. It is suggested that ELCs derived from BM-MSCs may be a endothelial progenitor cell source, which is able to re-endothelialize the injured artery during the early stages and attenuate neointima formation via intravenous transfusion.

A previous study identified that the majority of transplanted stem cells homed towards the lungs, and were retained in the lung alveoli via intravenous planting (22). The data indicated that BM-MSCs can home towards the injured artery, predominantly in the adventitia, with few 

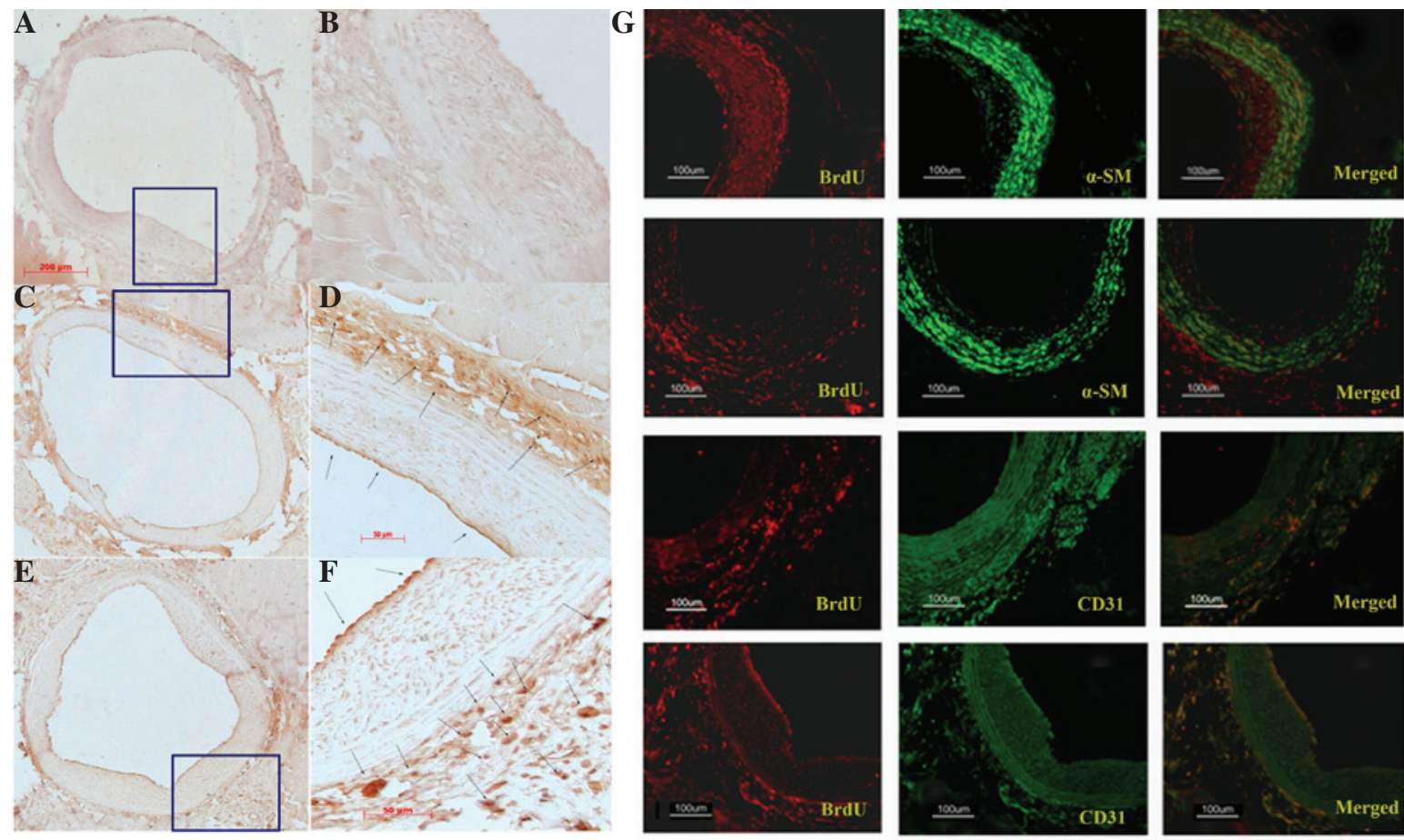

MSCs
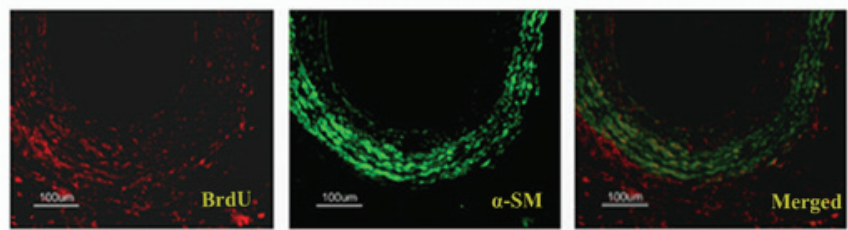

ELCs
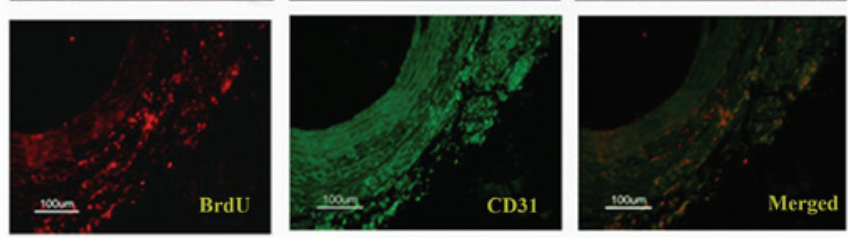

MSCs
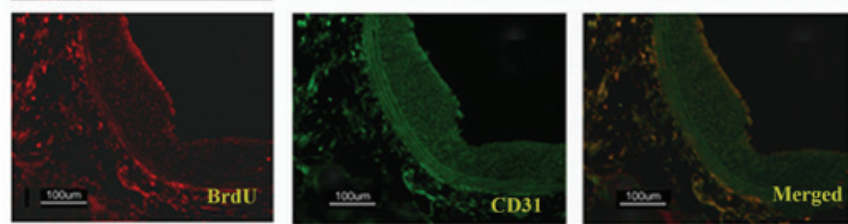

ELCs

Figure 3. (A and B) No BrdU+ cells were visible in the control injured artery vascular wall (A, x100; B, x400). (C-F) Immunohistochemistry staining with anti-BrdU highlighted numerous BrdU-labeling BM-MSCs (C, x100; D, x400) and ELCs (E, x100; F, x400) in the adventitia (arrow), particularly in the zone around the vasa vasorum, and few in the neointimal area (arrow) (F). (G) In the MSCs group, immunofluorescence analysis indicated that the majority of $\alpha$-SMA was expressed in the adventitia area, suggesting that MSCs differentiated into vascular smooth muscle cells. By contrast, few CD31+ transplanting cells were observed. In the ELCs group, numerous BrdU+-ELCs expressed CD31 in the surface and adventitia, indicating that ELCs homed to the injured artery and differentiated into endothelial cells. BrdU, 5-bromo-2-deoxyuridine; BM-MSCs, bone marrow-derived mesenchymal stem cells; ELCs, endothelial-like cells; $\alpha$-SMA; $\alpha$-smooth muscle actin.

in the intima and numerous cells in the vasa vasorum area, which was in agreement with a previous study (6). Notably, the majority of BM-MSCs and ELCs home towards the adventitia, and seldom MSCs and ELCs contribute to the neointima. Studies havew suggested that the arterial wall is a recipient and source of MSCs, and it has been demonstrated that a large population of vascular progenitor cells exist in the adventitia of the artery (23-25), which may be a niche of stem cells.

BM-MSCs were able to differentiate into ELCs in vitro. These cells presented with characteristics of endothelial markers however were not mature endothelial cells. Overall, the results suggested that transplantation not with BM-MSCs, however with ELCs, significantly suppressed intimal hyperplasia following vascular injury. These results indicate that ELCs differentiated from BM-MSCs can reduce neointimal formation subsequent to vascular lesions, which indicates important therapeutic implications for cardiovascular diseases and a new cell source for cell-based vascular engineering and repair in the future. Additional experiments are required in order to address the mechanisms of reduced neointimal formation by ELCs.

\section{Acknowledgements}

The current study was supported financially by grants from the Fund of Shanghai Municipal Commission of Health and Family Planning (grant no. 201440023) and Medical-Engineering Cross Fund (grant no. 2014128) of Shanghai Jiao
Tong University. The funders had no role in study design, data collection and analysis, decision to publish, or preparation of the manuscript.

\section{References}

1. Williams AR and Hare JM: Mesenchymal stem cells: Biology, pathophysiology, translational findings, and therapeutic implications for cardiac disease. Circ Res 109: 923-940, 2011.

2. Oswald J, Boxberger S, Jørgensen B, Feldmann S, Ehninger G, Bornhäuser $\mathrm{M}$ and Werner C: Mesenchymal stem cells can be differentiated into endothelial cells in vitro. Stem Cells 22: 377-384, 2004.

3. Silva GV, Litovsky S, Assad JA, Sousa AL, Martin BJ, Vela D, Coulter SC, Lin J, Ober J, Vaughn WK, et al: Mesenchymal stem cells differentiate into an endothelial phenotype, enhance vascular density, and improve heart function in a canine chronic ischemia model. Circulation 111: 150-156, 2005.

4. Suzuki S, Narita Y, Yamawaki A, Murase Y, Satake M, Mutsuga M, Okamoto H, Kagami H, Ueda M and Ueda Y: Effects of extracellular matrix on differentiation of human bone marrow-derived mesenchymal stem cells into smooth muscle cell lineage: Utility for cardiovascular tissue engineering. Cells Tissues Organs 191: 269-280, 2010.

5. Tang Z, Wang A, Yuan F, Yan Z, Liu B, Chu JS, Helms JA and Li S: Differentiation of multipotent vascular stem cells contributes to vascular diseases. Nat Commun 3: 875, 2012.

6. Forte A, Finicelli M, Mattia M, Berrino L, Rossi F, De Feo M, Cotrufo M, Cipollaro M, Cascino A and Galderisi U: Mesenchymal stem cells effectively reduce surgically induced stenosis in rat carotids. J Cell Physiol 217: 789-799, 2008.

7. Forte A, Rinaldi B, Sodano L, Berrino L, Rossi F, Finicelli M, Grossi M, Cobellis G, Botti C, De Feo M, et al: Stem cell therapy for arterial restenosis: Potential parameters contributing to the success of bone marrow-derived mesenchymal stromal cells. Cardiovasc Drugs Ther 26: 9-21, 2012. 
8. Shoji M, Oskowitz A,Malone CD, Prockop DJ and Pochampally R Human mesenchymal stromal cells (MSCs) reduce neointimal hyperplasia in a mouse model of flow-restriction by transient suppression of anti-inflammatory cytokines. J Atheroscler Thromb 18: 464-474, 2011

9. Feng J, Liu JP, Miao L, He GX, Li D, Wang HD and Jing T: Conditional expression of the type 2 angiotensin ii receptor in mesenchymal stem cells inhibits neointimal formation after arterial injury. J Cardiovasc Transl Res 7: 635-643, 2014

10. Wang CH, Cherng WJ, Yang NI, Kuo LT, Hsu CM, Yeh HI, Lan YJ, Yeh CH and Stanford WL: Late-outgrowth endothelial cells attenuate intimal hyperplasia contributed by mesenchymal stem cells after vascular injury. Arterioscler Thromb Vasc Biol 28: 54-60, 2008

11. Liao J, Chen X, Li Y, Ge Z, Duan H, Zou Y and Ge J: Transfer of bone-marrow-derived mesenchymal stem cells influences vascular remodeling and calcification after balloon injury in hyperlipidemic rats. J Biomed Biotechnol 2012: 165296, 2012.

12. Zhang FB, Li L, Fang B, Zhu DL, Yang HT and Gao PJ: Passage-restricted differentiation potential of mesenchymal stem cells into cardiomyocyte-like cells. Biochem Biophys Res Commun 336: 784-792, 2005.

13. Kim S, Kawamura M, Wanibuchi H, Ohta K, Hamaguchi A, Omura T, Yukimura T, Miura K and Iwao H: Angiotensin II type 1 receptor blockade inhibits the expression of immediate-early genes and fibronectin in rat injured artery. Circulation 92: 88-95, 1995.

14. Pankajakshan D, Kansal V and Agrawal DK: In vitro differentiation of bone marrow derived porcine mesenchymal stem cells to endothelial cells. J Tissue Eng Regen Med 7: 911-920, 2013.

15. Pittenger MF, Mackay AM, Beck SC, Jaiswal RK, Douglas R Mosca JD, Moorman MA, Simonetti DW, Craig S and Marshak DR: Multilineage potential of adult human mesenchymal stem cells. Science 284: 143-147, 1999.

16. Gan LM, Grönros J, Hägg U, Wikström J, Theodoropoulos C, Friberg $\mathrm{P}$ and Fritsche-Danielson R: Non-invasive real-time imaging of atherosclerosis in mice using ultrasound biomicroscopy. Atherosclerosis 190: 313-320, 2007.
17. Wu DJ, Xu JZ, Wu YJ, Jean-Charles L, Xiao B, Gao PJ and Zhu DL: Effects of fasudil on early atherosclerotic plaque formation and established lesion progression in apolipoprotein E-knockout mice. Atherosclerosis 207: 68-73, 2009.

18. Hillebrands JL, Klatter FA, van den Hurk BM, Popa ER, Nieuwenhuis $\mathrm{P}$ and Rozing J: Origin of neointimal endothelium and alpha-actin-positive smooth muscle cells in transplant arteriosclerosis. J Clin Invest 107: 1411-1422, 2001.

19. Grimm PC, Nickerson P, Jeffery J, Savani RC, Gough J, McKenna RM, Stern E and Rush DN: Neointimal and tubulointerstitial infiltration by recipient mesenchymal cells in chronic renal-allograft rejection. N Engl J Med 345: 93-97, 2001.

20. Li M, Li S, Yu L, Wu J, She T, Gan Y, Hu Z, Liao W and Xia H: Bone mesenchymal stem cells contributed to the neointimal formation after arterial injury. PLoS One 8: e82743, 2013.

21. Yue WM, Liu W, Bi YW, He XP, Sun WY, Pang XY, Gu XH and Wang XP: Mesenchymal stem cells differentiate into an endothelial phenotype, reduce neointimal formation, and enhance endothelial function in a rat vein grafting model. Stem Cells Dev 17: 785-793, 2008.

22. Kerkelä E, Hakkarainen T, Mäkelä T, Raki M, Kambur O, Kilpinen L, Nikkilä J, Lehtonen S, Ritamo I, Pernu R, et al: Transient proteolytic modification of mesenchymal stromal cells increases lung clearance rate and targeting to injured tissue. Stem Cells Transl Med 2: 510-520, 2013.

23. Psaltis PJ, Puranik AS, Spoon DB, Chue CD, Hoffman SJ, Witt TA, Delacroix S, Kleppe LS, Mueske CS, Pan S, et al: Characterization of a resident population of adventitial macrophage progenitor cells in postnatal vasculature. Circ Res 115: 364-375, 2014.

24. Chen Y, Wong MM, Campagnolo P, Simpson R, Winkler B, Margariti A, Hu Y and Xu Q: Adventitial stem cells in vein grafts display multilineage potential that contributes to neointimal formation. Arterioscler Thromb Vasc Biol 33: 1844-1851, 2013.

25. Grudzinska MK, Kurzejamska E, Bojakowski K, Soin J, Lehmann MH, Reinecke H, Murry CE, Soderberg-Naucler C and Religa P: Monocyte chemoattractant protein 1-mediated migration of mesenchymal stem cells is a source of intimal hyperplasia. Arterioscler Thromb Vasc Biol 33: 1271-1279, 2013. 